\title{
Social Networks: Prestige, Centrality, and Influence
}

\author{
Agnieszka Rusinowska ${ }^{1}$, Rudolf Berghammer ${ }^{2}$, Harrie De Swart ${ }^{3}$, and Michel \\ Grabisch $^{1}$ \\ 1 Centre d'Economie de la Sorbonne, Université Paris I Panthéon-Sorbonne \\ 106-112 Bd de l'Hôpital, 75647 Paris Cedex 13, France \\ \{agnieszka.rusinowska, michel.grabisch\}@univ-paris1.fr \\ 2 Institut für Informatik, Universität Kiel, Olshausenstraße 40, 24098 Kiel, Germany \\ rub@informatik. uni-kiel.de \\ 3 Department of Philosophy, Erasmus University Rotterdam \\ P.O. Box 1738, 3000 DR Rotterdam, The Netherlands; deSwart@fwb.eur.nl
}

\begin{abstract}
We deliver a short overview of different centrality measures and influence concepts in social networks, and present the relation-algebraic approach to the concepts of power and influence. First, we briefly discuss four kinds of measures of centrality: the ones based on degree, closeness, betweenness, and the eigenvector-related measures. We consider centrality of a node and of a network. Moreover, we give a classification of the centrality measures based on a topology of network flows. Furthermore, we present a certain model of influence in a social network and discuss some applications of relation algebra and RELVIEW to this model.
\end{abstract}

Keywords: social network, centrality, prestige, influence, relation algebra, RELVIEW

\section{Introduction}

Social networks play a central role in our activities, in social phenomena, in economic and political life. It is therefore crucial to provide an exhaustive analysis of social network structures and to study the impact they may have on human's behavior. Many scholars are particularly interested in measures that allow to compare networks. Also measures that compare nodes (representing agents) within a network and show how a node relates to the network are of interest. The question appears how central a node is and what its position and prestige in a network are. The concept of centrality as applied to human communication was introduced already in the late 1940's, and since then many different measures of centrality have been developed. They usually capture complementary aspects of a node's position, any hence a particular measure can be more appropriate for some applications and less for others.

One of the aims of this paper is to deliver a brief overview of the main centrality measures. Four kinds of measures are presented: degree centrality, closeness centrality, betweenness centrality, Katz prestige and Bonacich centrality. We also briefly discuss a categorization of centrality measures based on a topology of network flows. 
Social networks are particularly important in studying all kinds of influence phenomena. They are very useful for analyzing the diffusion of information and the formation of opinions and beliefs. It is therefore not surprising that there are numerous works in different scientific fields on the 'network approach' to interaction and influence.

One of the leading dynamic models on information transmission, opinion and consensus formation in networks is introduced by DeGroot [14]. Individuals start with initial opinions on a subject and put some weights on the current beliefs of other agents in forming their own beliefs for the next period. These beliefs are updated over time. Several variations and generalizations of the DeGroot model are presented e.g. in [15, 20,21, 22, 36]. Surveys of models of influence and different approaches to this phenomenon can be found e.g. in [27, 29, 36, 38].

Another framework of influence in networks is introduced in [33]. In the original one-step model, agents have to make their acceptance-rejection decision on a specific issue. Each agent has an inclination to say either 'yes' or 'no', but due to possible influence of the other agents, his final decision ('yes' or 'no') may be different from his initial inclination. This framework is extensively investigated e.g. in $[24,25,26,28,29,30,39]$.

Relation algebra is used very successfully for formal problem specification, prototyping, and algorithm development. For details on relations and relational algebra, see e.g. $[13,16,17,40]$. RELVIEW is a BDD-based tool for the visualization and manipulation of relations and for prototyping and relational programming. It has been developed at Kiel University. The tool is written in the $\mathrm{C}$ programming language and makes full use of the $\mathrm{X}$-windows graphical user interface. Details and applications can be found e.g. in $[3,4,9]$.

Several of our works are devoted to applications of relation algebra and RELVIEW to Game Theory and Social Choice Theory. In [5] we present such an application to coalition formation, where with the help of relation algebra and RELVIEW the set of all feasible stable governments is determined. A stable government is by definition not dominated by any other government. In [6] we deal with the case where all governments are dominated. By using notions from relation algebra, graph theory and social choice theory, and by using RELVIEw we can compute a government that is as close as possible to being non-dominated. In [7] we apply relation algebra and RELVIEW to networks, i.e., to compute some measures of agents' strength in a network, like power, success, and influence. In [8] we present relation-algebraic models of simple games and develop relational specifications for solving some basic game-theoretic problems. We test fundamental properties of simple games, compute specific players and coalitions, and apply relation algebra to determine power indices.

In this paper we also aim at presenting a relation-algebraic approach to the concepts of influence in a social network. We recapitulate relation-algebraic specifications (presented in [7]) of the following concepts of the model of influence $([25,33,39])$ : the inclination and decision vectors, the group decision, the HoedeBakker index, the inclination vectors of potential and observed influence, and the set of followers. 
The paper is structured as follows. In Section 2 the basic concepts in network theory are recalled. In Section 3 we discuss the main centrality measures. Section 4 concerns the model of influence in a social network. In Section 5 the relation-algebraic preliminaries are presented. Section 6 is devoted to the relation-algebraic approach to the concepts of influence. In Section 7 we present some concluding remarks.

\section{The basic concepts in network theory}

In this section we present the preliminaries on networks. For textbooks on network theory, see e.g. [23, 36, 44].

Let $N=\{1,2, \ldots, n\}$ be a (finite) set of nodes. By $g_{i j} \in\{0,1\}$ we denote a relationship between nodes $i$ and $j$, where

$$
g_{i j}= \begin{cases}1 & \text { if there is a link between } i \text { and } j \\ 0 & \text { otherwise. }\end{cases}
$$

In what follows we only consider undirected links, i.e., we assume that $g_{i j}=g_{j i}$.

A network $g$ is defined as a set of nodes $N$ with links between them. Let $\mathcal{G}$ denote the collection of all possible networks on $n$ nodes.

By $N_{i}(g)$ we denote the neighborhood (the set of neighbors) of node $i$ in network $g$, i.e., the set of nodes with which node $i$ has a link:

$$
N_{i}(g)=\left\{j \in N: g_{i j}=1\right\} .
$$

The degree $d_{i}(g)$ of a node $i$ in $g$ is the number of $i$ 's neighbors in $g$, i.e.,

$$
d_{i}(g)=\left|N_{i}(g)\right| .
$$

A network $g$ is said to be regular if every node has the same number of neighbors, i.e., if for some $d \in\{0,1, \ldots, n-1\}, d_{i}(g)=d$ for each $i \in N$.

A complete network is a regular network with $d=n-1$. The empty network is a regular network with $d=0$.

One of the concerns when analyzing a network is to check how one node may be reached from another one. We distinguish between the following definitions:

- A walk is a sequence of nodes in which two nodes have a link (they are neighbors), and a node or a link may appear more than once. Its length is simply the number of links in the walk.

- A trail is a walk in which all links are distinct.

- A path is a trail in which all nodes are distinct.

- A cycle is a trail with at least 3 nodes in which the initial node and the end node are the same.

- A geodesic between two nodes is a shortest path between them.

If there is a path between $i$ and $j$ in $g$, then the geodesic distance $d(i, j ; g)$ between these two nodes $i$ and $j$ is therefore equal to

$$
d(i, j ; g)=\text { the number of links in a shortest path between } i \text { and } j .
$$


If there is no path between $i$ and $j$ in $g$, we set $d(i, j ; g)=\infty$.

A star is a network in which there exists some node $i$ (referred to as the center of the star) such that every link in the network involves node $i$.

Two nodes belong to the same component if and only if there exists a path between them. A network is connected if there exists a path between any pair of nodes $i, j \in N$. Consequently, a network is connected if and only if it consists of a single component.

The adjacency matrix $\mathbf{G}$ of a (undirected or directed) network $g$ is defined as $\mathbf{G}=\left[g_{i j}\right]$ with $g_{i j}$ as in (1). In other words, an entry in the matrix $\mathbf{G}$ corresponding to the pair $\{i, j\}$ signifies the presence or absence of a link between $i$ and $j$. Let $\mathbf{G}^{k}$ denote the $k$ th power of $\mathbf{G}$, i.e., $\mathbf{G}^{k}=\left[g_{i j}^{k}\right]$, where $g_{i j}^{k}$ measures the number of walks of length $k$ that exist between $i$ and $j$ in network $g$. We have $\mathbf{G}^{0}=\mathbf{I}$, where $\mathbf{I}$ is the $n \times n$ identity matrix.

\section{Different measures of centrality in networks}

The concept of centrality captures a kind of prominence of a node in a network. The economic and sociological literature offers several such concepts. For surveys of different notions of centrality, see e.g. [19, 23, 36]. In this paper, we recapitulate several well-known centrality measures. The presentation is based on the three references mentioned above.

As presented in [36], measures of centrality can be categorized into the following main groups:

(1) Degree centrality

(2) Closeness centrality

(3) Betweenness centrality

(4) Prestige- and eigenvector-related centrality.

\subsection{Degree centrality}

The degree centrality indicates how well a node is connected in terms of direct connections, i.e., it keeps track of the degree of the node. This measure can be seen as an index of the node's communication activity.

The degree centrality $C_{d}(i ; g)$ of node $i$ in network $g$ is given by

$$
C_{d}(i ; g)=\frac{d_{i}(g)}{n-1}=\frac{\left|N_{i}(g)\right|}{n-1}
$$

where $N_{i}(g)$ and $d_{i}(g)$ are defined in (2) and (3). Obviously, $0 \leq C_{d}(i ; g) \leq 1$.

Let $i^{*}$ be a node which attains the highest degree centrality $C_{d}\left(i^{*} ; g\right)$ in $g$. The degree centrality $C_{d}(g)$ of network $g$ is given by

$$
C_{d}(g)=\frac{\sum_{i=1}^{n}\left[C_{d}\left(i^{*} ; g\right)-C_{d}(i ; g)\right]}{\max _{g^{\prime} \in \mathcal{G}}\left[\sum_{i=1}^{n}\left[C_{d}\left(i^{*} ; g^{\prime}\right)-C_{d}\left(i ; g^{\prime}\right)\right]\right]} .
$$


Since the minimum degree is 1 and the maximum degree is $(n-1)$, one can easily see that the denominator of (6) is equal to $\frac{(n-2)(n-1)}{(n-1)}$, and hence

$$
C_{d}(g)=\frac{\sum_{i=1}^{n}\left[C_{d}\left(i^{*} ; g\right)-C_{d}(i ; g)\right]}{n-2} .
$$

Note that $C_{d}(g)=1$ if $g$ is a star, and $C_{d}(g)=0$ if $g$ is a regular network.

\subsection{Closeness centrality}

The closeness centrality is based on proximity and measures how easily a node can reach other nodes in a network. It is a kind of a measure of the node's independence or efficiency.

The closeness centrality $C_{c}(i ; g)$ of node $i$ in network $g$ is defined as

$$
C_{c}(i ; g)=\frac{n-1}{\sum_{j \neq i} d(i, j ; g)}
$$

where $d(i, j ; g)$ is the geodesic distance between $i$ and $j$ as defined in (4), and $(n-1)$ is the minimum possible total distance from $i$ to all other nodes in $g$. There is a whole family of closeness measures [44] based on different conventions for dealing with non-connected networks and other possible measures of distance.

Let $i^{*}$ be a node which attains the highest closeness centrality $C_{c}\left(i^{*} ; g\right)$ in $g$. The closeness centrality $C_{c}(g)$ of network $g$ is given by

$$
C_{c}(g)=\frac{\sum_{i=1}^{n}\left[C_{c}\left(i^{*} ; g\right)-C_{c}(i ; g)\right]}{\max _{g^{\prime} \in \mathcal{G}}\left[\sum_{i=1}^{n}\left[C_{c}\left(i^{*} ; g^{\prime}\right)-C_{c}\left(i ; g^{\prime}\right)\right]\right]} .
$$

One can show (see e.g. [19]) that

$$
C_{c}(g)=\frac{\sum_{i=1}^{n}\left[C_{c}\left(i^{*} ; g\right)-C_{c}(i ; g)\right]}{(n-2)(n-1) /(2 n-3)} .
$$

Note that $C_{c}(g)=1$ if $g$ is a star, and $C_{c}(g)=0$ if $g$ is a cycle. Obviously, although $C_{d}(g)=C_{c}(g)$ for $g$ being a star or a cycle, in general $C_{d}(g) \neq C_{c}(g)$.

\subsection{Betweenness centrality}

The betweenness centrality (introduced in [18]) is based on how important a node is in terms of connecting other nodes. It is useful as an index of the potential of a node for control of communication.

By $P_{i}(k j)$ and $P(k j)$ we denote the number of geodesics between $k$ and $j$ containing $i \notin\{k, j\}$, and the total number of geodesics between $k$ and $j$, respectively.

The betweenness centrality $C_{b}(i ; g)$ of node $i$ in network $g$ is defined as

$$
C_{b}(i ; g)=\frac{2}{(n-1)(n-2)} \sum_{k \neq j: i \notin\{k, j\}} \frac{P_{i}(k j)}{P(k j)} .
$$


Note that $\frac{P_{i}(k j)}{P(k j)}$ is the probability that $i$ falls on a randomly selected geodesic linking $k$ and $j$, and the number of all pairs of nodes (different from $i$ ) is equal to $\left(\begin{array}{c}n-1 \\ 2\end{array}\right)=\frac{(n-1)(n-2)}{2}$. In particular, if $g$ is a star, then $C_{b}(i ; g)=1$ for $i$ being the center and $C_{b}(i ; g)=0$ otherwise.

Let $i^{*}$ be a node which attains the highest betweenness centrality $C_{b}\left(i^{*} ; g\right)$ in $g$. The betweenness centrality $C_{b}(g)$ of network $g$ is given by

$$
C_{b}(g)=\frac{\sum_{i=1}^{n}\left[C_{b}\left(i^{*} ; g\right)-C_{b}(i ; g)\right]}{n-1} .
$$

\subsection{Prestige- and eigenvector-related centrality measures}

There exist other measures of centrality that take into account a richer range of direct and indirect influences in networks. The measures developed e.g. in $[10,11,37]$ are based on the idea that a node's importance is determined by the importance of its neighbors.

The Katz prestige $P_{i}^{K}(g)$ of node $i$ in $g$ is defined as

$$
P_{i}^{K}(g)=\sum_{j \neq i} g_{i j} \frac{P_{j}^{K}(g)}{d_{j}(g)} .
$$

This means that the Katz prestige of $i$ is equal to the sum of the prestiges of $i$ 's neighbors divided by their respective degrees. In other words, the measure is corrected by the number of neighbors of node $j$ (if $j$ has more relationships, then $i$ gets less prestige from being connected to $j$ ). Note that this definition is self-referential. (11) can be rewritten as

$$
\begin{aligned}
& \mathbf{P}^{K}(g)=\mathbf{G}^{\prime} \mathbf{P}^{K}(g) \\
& \left(\mathbf{I}-\mathbf{G}^{\prime}\right) \mathbf{P}^{K}(g)=\mathbf{0}
\end{aligned}
$$

where $\mathbf{P}^{K}(g)$ is the $n \times 1$ vector of $P_{i}^{K}(g), i \in N, \mathbf{I}$ is the $n \times n$ identity matrix, and $\mathbf{G}^{\prime}=\left[g_{i j}^{\prime}\right]$ is the normalized adjacency matrix with $g_{i j}^{\prime}=\frac{g_{i j}}{d_{j}(g)}$. In other words, calculating the Katz prestige is reduced to finding the unit eigenvector of $\mathbf{G}^{\prime}$. Obviously, $\mathbf{P}^{K}(g)$ is determined up to a scale factor.

Katz [37] introduced another measure of prestige, where the prestige of a node is a weighted sum of the walks that emanate from it, and a walk of length

$k$ is worth $a^{k}$, for some parameter $0<a<1$. The second prestige measure of Katz is given by

$$
\mathbf{P}^{K 2}(g, a)=(\mathbf{I}-a \mathbf{G})^{-1} a \mathbf{G} \mathbf{1}
$$

where 1 is the $n \times 1$ vector of $1 \mathrm{~s}$, and $a$ is sufficiently small.

The Bonacich centrality is an extension of the second prestige measure of Katz and is expressed by

$$
\mathbf{C}^{B}(g, a, b)=(\mathbf{I}-b \mathbf{G})^{-1} a \mathbf{G} \mathbf{1}
$$

where $a>0$ and $b>0$ are scalars, and $b$ is sufficiently small. 


\subsection{Categorizing centrality measures by a topology of network flows}

The relation between the major centrality measures and different flow processes is extensively discussed in [12]. Centrality measures make implicit assumptions about network flow, and hence they are matched to the kinds of flows they are appropriate for.

The typology of network flows is based on two dimensions:

- the trajectory dimension - kinds of trajectories that traffic may follow: geodesics, paths, trails, walks;

- the transmission dimension - methods of spread: parallel (simultaneous) duplication, serial (once at a time) duplication, transfer.

Table 1 classifies different kinds of traffic based on these two dimensions.

Table 1. Topology of flow processes (see [12])

\begin{tabular}{c|c|c|c} 
& parallel duplication & serial duplication & transfer \\
\hline geodesics & - & mitotic reproduction & package delivery \\
paths & internet name-server & viral infection & mooch \\
trails & e-mail broadcast & gossip & used goods \\
walks & attitude influencing & emotional support & money exchange
\end{tabular}

Table 2 classifies the major centrality measures presented above, based on flow processes.

Table 2. Flow processes and major centrality measures (see [12])

\begin{tabular}{c|c|c|c} 
& parallel duplication & serial duplication & transfer \\
\hline geodesics & & closeness & closeness \\
paths & closeness, degree & & \\
trails & closeness, degree & & \\
walks & closeness, degree & & \\
& Bonacich eigenvector & & \\
& Katz prestige & &
\end{tabular}

Since each centrality measure is appropriate for particular kinds of flows, applying these measures to other flow processes that they are not designed for leads to wrong results. For example, one can use the closeness and betweenness centrality measures for package delivery, but it is inappropriate to use them to indicate who will receive news early in a gossip. For a discussion on this classification, see [12]. 


\section{The model of influence in a social network}

In this section we present a framework of influence originally introduced in [33] and refined in $[25,39]$.

\subsection{The Hoede-Bakker index}

We consider a social network with a set of agents (players, actors, voters) denoted by $N:=\{1,2, \ldots, n\}$ who are to make a certain acceptance-rejection decision on a specific proposal. Each agent $k \in N$ has an inclination $i_{k}$ either to say 'yes' (denoted by +1 ) or 'no' (denoted by -1$)$. Let $i=\left(i_{1}, i_{2}, \ldots, i_{n}\right)$ denote an inclination vector and $I:=\{-1,+1\}^{n}$ be the set of all inclination vectors.

It is assumed that agents may influence each other, and due to the influences, the final decision of an agent may be different from his original inclination. Formally, each inclination vector $i \in I$ is transformed into a decision vector $B i=\left((B i)_{1},(B i)_{2}, \ldots,(B i)_{n}\right)$, where $B: I \rightarrow I, i \mapsto B i$ is the influence function. Let $B(I)$ be the set of all decision vectors under $B$ and let $\mathcal{B}$ denote the set of all influence functions.

We also assume a group decision function $g d: B(I) \rightarrow\{-1,+1\}$, having the value +1 if the group decision is 'yes', and the value -1 if the group decision is 'no'. The set of all group decision functions will be denoted by $\mathcal{G}$.

In [39] we introduce the following generalized index. Given $B \in \mathcal{B}$ and $g d \in \mathcal{G}$, the generalized Hoede-Bakker index of player $k \in N$ is defined as

$$
\operatorname{GHB}_{k}(B, g d):=\frac{\left|I_{k}^{++}\right|-\left|I_{k}^{+-}\right|+\left|I_{k}^{--}\right|-\left|I_{k}^{-+}\right|}{2^{n}}
$$

where

$$
\begin{aligned}
& I_{k}^{++}:=\left\{i \in I \mid i_{k}=+1 \wedge g d(B i)=+1\right\} \\
& I_{k}^{+-}:=\left\{i \in I \mid i_{k}=+1 \wedge g d(B i)=-1\right\} \\
& I_{k}^{--}:=\left\{i \in I \mid i_{k}=-1 \wedge g d(B i)=-1\right\} \\
& I_{k}^{-+}:=\left\{i \in I \mid i_{k}=-1 \wedge g d(B i)=+1\right\} .
\end{aligned}
$$

Obviously all the four sets depend on $(B, g d)$, which has been skipped for convenience of notation.

Note that the generalized Hoede-Bakker index, although defined in the influence setup, does not measure any influence. As remarked in [39] the GHB index is a kind of 'net Success', i.e., 'Success - Failure'.

\subsection{The influence indices}

Measures of influence, the so called influence indices, are defined in [25]. Below we recall these definitions.

Concerning notation, for convenience we omit braces for sets, e.g., $N \backslash\{j\}$ is written as $N \backslash j$. For any $S \subseteq N,|S| \geq 2$, we introduce the set $I_{S}$ of all inclination vectors in which all members of $S$ have the same inclination

$$
I_{S}:=\left\{i \in I \mid \forall k, j \in S\left[i_{k}=i_{j}\right]\right\}
$$


and $I_{k}:=I$, for any $k \in N$. For $i \in I_{S}$ we denote by $i_{S}$ the value $i_{k}$ for some $k \in S$. Let for each $S \subseteq N$ and $j \in N \backslash S, I_{S \rightarrow j}$ denote the set of all inclination vectors of potential influence of $S$ on $j$, that is,

$$
I_{S \rightarrow j}:=\left\{i \in I_{S} \mid i_{j}=-i_{S}\right\} .
$$

Moreover, for each $B \in \mathcal{B}$, let $I_{S \rightarrow j}^{*}(B)$ denote the set of all inclination vectors of observed influence of $S$ on $j$ under $B \in \mathcal{B}$, that is,

$$
I_{S \rightarrow j}^{*}(B):=\left\{i \in I_{S \rightarrow j} \mid(B i)_{j}=i_{S}\right\} .
$$

In [25] we introduce the weighted influence indices, whose main idea is to give a relative importance to the different inclination vectors. For each $S \subseteq N$, $j \in N \backslash S$ and $i \in I_{S}$, we introduce a weight $\alpha_{i}^{S \rightarrow j} \in[0,1]$ of influence of coalition $S$ on $j \in N \backslash S$ under the inclination vector $i \in I_{S}$. There is no normalization on the weights, but we assume that for each $S \subseteq N$ and $j \in N \backslash S$, there exists $i \in I_{S \rightarrow j}$ such that $\alpha_{i}^{S \rightarrow j}>0$.

Given $B \in \mathcal{B}$, for each $S \subseteq N, j \in N \backslash S$, the weighted influence index of coalition $S$ on player $j$ is defined as

$$
d_{\alpha}(B, S \rightarrow j):=\frac{\sum_{i \in I_{S \rightarrow j}^{*}(B)} \alpha_{i}^{S \rightarrow j}}{\sum_{i \in I_{S \rightarrow j}} \alpha_{i}^{S \rightarrow j}} \in[0,1] .
$$

It is the (weighted) proportion of situations of observed influence among all situations of potential influence. Two particular ways of weighting lead to the possibility influence index $\bar{d}(B, S \rightarrow j)$ and the certainty influence index $\underline{d}(B, S \rightarrow j)$. We have for each $S \subseteq N, j \in N \backslash S$ and $B \in \mathcal{B}$

$$
\bar{d}(B, S \rightarrow j)=d_{\bar{\alpha}}(B, S \rightarrow j), \text { where } \bar{\alpha}_{i}^{S \rightarrow j}=1 \text { for each } i \in I_{S}
$$

and

$$
\begin{aligned}
\underline{d}(B, S \rightarrow j) & =d_{\underline{\alpha}}(B, S \rightarrow j), \text { where for each } i \in I_{S} \\
\underline{\alpha}_{i}^{S \rightarrow j} & = \begin{cases}1, & \text { if } \forall p \notin S \cup j, i_{p}=-i_{S} \\
0, & \text { otherwise. }\end{cases}
\end{aligned}
$$

Consequently, we have

$$
\begin{gathered}
\bar{d}(B, S \rightarrow j)=\frac{\left|I_{S \rightarrow j}^{*}(B)\right|}{\left|I_{S \rightarrow j}\right|} \in[0,1] \\
\underline{d}(B, S \rightarrow j)=\frac{\left|\left\{i \in I_{S \rightarrow j}^{*}(B) \mid \forall p \notin S\left[i_{p}=-i_{S}\right]\right\}\right|}{2} \in\left\{0, \frac{1}{2}, 1\right\} .
\end{gathered}
$$

The possibility influence index gives therefore the fraction of potential influence situations that happen to be situations of observed influence indeed. The certainty influence index measures also such a fraction, except that it focuses only on situations in which the coalition in question is the only one which influences the agent. 


\subsection{Followers and kernel}

The key concept of the influence framework is the concept of follower of a given coalition, that is, an agent who always follows the inclination of that coalition when all members of the coalition have the same inclination. The follower function of $B \in \mathcal{B}$ is a mapping $F_{B}: 2^{N} \rightarrow 2^{N}$ defined as

$$
F_{B}(S):=\left\{k \in N \mid \forall i \in I_{S},(B i)_{k}=i_{S}\right\}, \quad \forall S \subseteq N, S \neq \emptyset
$$

and $F_{B}(\emptyset):=\emptyset$. We say that $F_{B}(S)$ is the set of followers of $S$ under $B$. The set of all follower functions is denoted by $\mathcal{F}$. In [25] it is shown that

$$
d_{\alpha}(B, S \rightarrow j)=1, \quad \forall j \in F_{B}(S) \backslash S .
$$

Another important concept of the influence model is the concept of kernel of an influence function, which is the set of 'truly' influential coalitions. Assume $F_{B}$ is not identical to the empty set. The kernel of $B$ is defined as

$$
\mathcal{K}(B):=\left\{S \in 2^{N} \mid F_{B}(S) \neq \emptyset \text {, and } S^{\prime} \subset S \Rightarrow F_{B}\left(S^{\prime}\right)=\emptyset\right\} .
$$

In [25] we also define some specific influence functions and study their properties, e.g., the sets of followers and kernels of these functions.

\subsection{Further research on influence}

The model of influence presented above, i.e., the model of initial inclinations and final decisions, is studied extensively in several other works:

- In [26] we generalize the basic yes-no model of influence to a framework in which every agent has a totally ordered set of possible actions, the same for each player, and he has an inclination to choose a particular action. We investigate the generalized influence indices, different influence functions, and other tools related to the influence in the multi-choice model.

- In [28] we consider the influence model with a continuum of actions. In this generalized framework we introduce and study measures of positive and negative influence and other tools for analyzing influence. Also the set of fixed points under a given influence function is analyzed. Furthermore, we study linear influence functions.

- The results presented in [24] concern a comparison of the influence model with the framework of command games [34,35]. We show that the framework of influence is more general than the framework of the command games. In particular, we define several influence functions which capture the command structure. For some influence functions we define the equivalent command games.

- In [30] we establish the exact relations between the key concepts of the influence model and the framework of command games. We deliver sufficient and necessary conditions for a function to be a follower function, and describe the structure of the set of all influence functions that lead to a given follower 
function. We also deliver sufficient and necessary conditions for a function to be a command function, and describe the minimal sets generating a normal command game. In addition, we study the relation between command games and influence functions.

- We also study the dynamics of influence. In [29] the yes-no model with a single step of mutual influence is generalized to a framework with iterated influence. We analyze the decision process in which the mutual influence does not stop after one step but iterates, and we study the convergence of an influence function. In particular, we investigate stochastic influence functions and apply the theory of Markov chains to the analysis of such functions. Moreover, we propose a general framework of influence based on aggregation functions.

\section{$5 \quad$ Relation-algebraic preliminaries}

In this section we present the basics of relation algebra.

If $X$ and $Y$ are sets, then a subset $R$ of the Cartesian product $X \times Y$ is called a (binary) relation with domain $X$ and range $Y$. We denote the set (also called type) of all relations with domain $X$ and range $Y$ by $[X \leftrightarrow Y]$ and write $R: X \leftrightarrow Y$ instead of $R \in[X \leftrightarrow Y]$. If $X$ and $Y$ are finite sets of size $m$ and $n$ respectively, then we may consider a relation $R: X \leftrightarrow Y$ as a Boolean matrix with $m$ rows and $n$ columns and entries from $\{0,1\}$. The Boolean matrix interpretation of relations is used as one of the graphical representations of relations within the RELVIEW tool. We can speak about rows, columns and entries of a relation and write $R_{x, y}$ instead of $\langle x, y\rangle \in R$ or $x R y$.

The basic operations on relations are $R^{\top}$ (transposition, conversion), $\bar{R}$ (complement, negation), $R \cup S$ (union, join), $R \cap S$ (intersection, meet), $R S$ (composition, multiplication), and the special relations $\mathrm{O}$ (empty relation), $\mathrm{L}$ (universal relation), and I (identity relation). If $R$ is included in $S$ we write $R \subseteq S$, and equality of $R$ and $S$ is denoted as $R=S$.

A membership relation $\mathrm{E}: X \leftrightarrow 2^{X}$ relates $x \in X$ and $Y \in 2^{X}$ iff $x \in Y$.

The expression $\operatorname{syq}(R, S):=\overline{R^{\top} \bar{S}} \cap \overline{\bar{R}^{\top} S}$ is by definition the symmetric quotient $\operatorname{syq}(R, S): Y \leftrightarrow Z$ of two relations $R: X \leftrightarrow Y$ and $S: X \leftrightarrow Z$. Many properties of this construct can be found e.g. in [40]. In particular, for all $y \in Y$ and $z \in Z$ the relationship $\operatorname{syq}(R, S)_{y, z}$ holds iff for all $x \in X$ the equivalence $R_{x, y} \leftrightarrow S_{x, z}$ is true, i.e., if the $y$-column of $R$ and the $z$-column of $S$ coincide.

Given a Cartesian product $X \times Y$ of two sets $X$ and $Y$, there are two projection functions which decompose a pair $u=\left(u_{1}, u_{2}\right)$ into its first component $u_{1}$ and its second component $u_{2}$. For a relation-algebraic approach it is useful to consider the corresponding projection relations $\pi: X \times Y \leftrightarrow X$ and $\rho: X \times Y \leftrightarrow Y$ such that for all pairs $u \in X \times Y$ and elements $x \in X$ and $y \in Y$ we have $\pi_{u, x}$ iff $u_{1}=x$ and $\rho_{u, y}$ iff $u_{2}=y$.

Projection relations enable us to describe the well-known pairing operation of functional programming relation-algebraically as follows: For relations $R$ : 
$Z \leftrightarrow X$ and $S: Z \leftrightarrow Y$ we define their pairing (frequently also called fork or tupling) $[R, S]: Z \leftrightarrow X \times Y$ by $[R, S]:=R \pi^{\top} \cap S \rho^{\top}$. Then for all $z \in Z$ and pairs $u=\left(u_{1}, u_{2}\right) \in X \times Y$ a simple reflection shows that $[R, S]_{z, u}$ iff $R_{z, u_{1}}$ and $S_{z, u_{2}}$.

Column vectors are relations $v$ with $v=v \mathrm{~L}$. As for a column vector the range is irrelevant, we consider only vectors $v: X \leftrightarrow \mathbf{1}$ with a specific singleton set $\mathbf{1}:=\{\perp\}$ as range. A column vector $v: X \leftrightarrow \mathbf{1}$ can be considered as a Boolean matrix with exactly one column, i.e., as a Boolean column vector, and it describes the subset $\left\{x \in X \mid v_{x, \perp}\right\}$ of its domain $X$. If $v: X \leftrightarrow \mathbf{1}$ describes the subset $S$ of $X$ in the sense above, then the injective mapping $\operatorname{inj}(v): S \leftrightarrow X$ is obtained from the identity relation I $: X \leftrightarrow X$ by removing all rows which correspond to a 0 -entry in $v$. Hence, we have $\operatorname{inj}(v)_{j, k}$ iff $j=k$.

A non-empty column vector $v$ is a column point if $v v^{\top} \subseteq$ I, i.e., it is injective in the relational sense. In the Boolean matrix model, a column point $v: X \leftrightarrow \mathbf{1}$ is a Boolean column vector in which exactly one entry is 1 .

Vectors also allow to formalize the notions of $y$-columns and $x$-rows. For a relation $R: X \leftrightarrow Y$ and $y \in Y$, the column vector $v: X \leftrightarrow \mathbf{1}$ equals the $y$-column of $R$ if for all $x \in X$ we have $v_{x, \perp}$ iff $R_{x, y}$.

Row vectors are relations defined as the transposes of column vectors. We only need row vectors $v$ of the specific type $[\mathbf{1} \leftrightarrow Y]$ that correspond to Boolean row vectors. Then $v$ describes the subset $\left\{y \in Y \mid v_{\perp, y}\right\}$ of its range $Y$.

If $v: 2^{M} \leftrightarrow \mathbf{1}$ represents the subset $\mathcal{S}$ of $2^{M}$ and the size of the domain of $w: W \leftrightarrow \mathbf{1}$ is at most $|M|+1$, then for all $X \in 2^{M}$ we have $\operatorname{cardfilter}(v, w)_{X, \perp}$ iff $X \in \mathcal{S}$ and $|X|<|W|$. Hence, the complement of cardfilter $(\mathrm{L}, w)$ represents the subset of $2^{M}$ whose elements have at least size $|W|$.

\section{Applying relation algebra to the model of influence}

In this section we deal with the relation-algebraic approach to the model of influence in a social network. We recall some selected results presented in [7].

\subsection{Modeling the inclination and decision vectors}

For modeling inclination vectors and decision vectors, we use column vectors. For modeling subsets of the sets $I$ and $B(I)$, we use row vectors.

We assume a social network with a set $N$ of players. Let $D: N \leftrightarrow N$ be the relation of the dependency graph of the network. This means that there is an arc from an agent $j \in N$ to an agent $k \in N$ iff $D_{j, k}$ holds. Then the set of the dependent agents is described relation-algebraically by the column vector

$$
\operatorname{depend}(D):=D^{\top} \mathrm{L}
$$

of type $[N \leftrightarrow \mathbf{1}]$, where $\mathbf{L}$ has type $[N \leftrightarrow \mathbf{1}]$ as well.

The set $I$ of all inclination vectors can immediately be modeled by the columns of the membership relation $\mathrm{E}: N \leftrightarrow 2^{N}$. Hence, we regard inclination vectors and the corresponding decision vectors as relational column vectors $i: N \leftrightarrow \mathbf{1}$ and $B i: N \leftrightarrow \mathbf{1}$, respectively. 
We develop a column-wise enumeration of the set $B(I)$ of decision vectors with relation-algebraic means. The influence function $B$ is given by the rule 'following only unanimous trend-setters', which means that an agent follows his trend setters only if they all have the same inclination. In [7] we prove that:

Theorem 6.1 For each inclination vector $i: N \leftrightarrow \mathbf{1}$, the decision vector $B i$ : $N \leftrightarrow \mathbf{1}$ under the rule 'following only unanimous trend-setters' is given by

$$
B i=\left(i \cap\left(\bar{d} \cup\left(d \cap D^{\top} i \cap D^{\top} \bar{i}\right)\right)\right) \cup\left(d \cap \overline{D^{\top} \bar{i}}\right),
$$

where $d:=\operatorname{depend}(D)$.

The relation-algebraic expression $\left(i \cap\left(\bar{d} \cup\left(d \cap D^{\top} i \cap D^{\top} \bar{i}\right)\right)\right) \cup\left(d \cap \overline{D^{\top} \bar{i}}\right)$ is built from $i$ using unions, intersections, complements and left-compositions with constants only. If we replace the column vector $i: N \leftrightarrow \mathbf{1}$ by the membership relation $\mathrm{E}: N \leftrightarrow 2^{N}$ that column-wisely enumerates all inclination vectors and adapt simultaneously the type $[N \leftrightarrow \mathbf{1}]$ of $d$ to the type $\left[N \leftrightarrow 2^{N}\right]$ of $\mathrm{E}$ by a right-composition with the universal row vector $L: \mathbf{1} \leftrightarrow 2^{N}$, we get the relation

$$
\operatorname{Dvec}(D):=\left(\mathrm{E} \cap\left(\overline{d \mathrm{~L}} \cup\left(d \mathrm{~L} \cap D^{\top} \mathrm{E} \cap D^{\top} \overline{\mathrm{E}}\right)\right)\right) \cup\left(d \mathrm{~L} \cap \overline{D^{\top} \overline{\mathrm{E}}}\right)
$$

of type $\left[N \leftrightarrow 2^{N}\right]$ that column-wisely enumerates the set $B(I)$ of decision vectors.

\subsection{Computing the group decisions}

Next, we deliver a relation-algebraic specification of the group decisions under majority as decision rule via a row vector.

We assume that a row vector $m: \mathbf{1} \leftrightarrow 2^{N}$ is available such that for all $X \in 2^{N}$ we have $m_{\perp, X}$ iff $|X| \geq\left[\frac{|N|}{2}\right]+1$. In RELVIEW such a vector can be easily obtained with the help of the base operation cardfilter as

$$
m:=\overline{\operatorname{cardfilter}(\mathrm{L}, w)}^{\mathrm{\top}},
$$

where the first argument $L: 2^{N} \leftrightarrow \mathbf{1}$ describes the entire powerset $2^{N}$, and the second argument $w: W \leftrightarrow \mathbf{1}$ determines the threshold for majority by its length, i.e., fulfills $|W|=\left[\frac{|N|}{2}\right]+1$. In [7] we show the following result:

Theorem 6.2 Let, based on the specifications (24) and (25), the row vector $g d v(D)$ of type $\left[\mathbf{1} \leftrightarrow 2^{N}\right]$ be defined by

$$
g d v(D):=m \operatorname{syq}(\mathrm{E}, \operatorname{Dvec}(D)),
$$

where $\mathrm{E}: N \leftrightarrow 2^{N}$ is the membership relation. Then we have for all $X \in 2^{N}$ : If the decision vector $B i: N \leftrightarrow \mathbf{1}$ equals the $X$-column of Dvec $(D)$, then $g d v(D)_{\perp, X}$ holds iff the number of 1 -entries in $B i$ is at least $\left[\frac{|N|}{2}\right]+1$. 


\subsection{Computing the Hoede-Bakker index}

We assume that the player $k \in N$, on which the sets $I_{k}^{++}, I_{k}^{+-}, I_{k}^{-+}$and $I_{k}^{--}$ depend, is described by a column point $p: N \leftrightarrow \mathbf{1}$ in the relational sense. As the definitions of the sets use the values $g d(B i)$ for $i \in I$, we assume that the group decision row vector $g:=g d v(D)$ is at hand. In [7] we prove the following:

Theorem 6.3 Let, depending on the column point $p: N \leftrightarrow \mathbf{1}$ and the row vector $g: \mathbf{1} \leftrightarrow 2^{N}$, the four vectors ipp $(p, g)$, ipm $(p, g)$, imp $(p, g)$ and imm $(p, g)$ of type $\left[\mathbf{1} \leftrightarrow 2^{N}\right]$ be defined as follows, where $\mathrm{E}: N \leftrightarrow 2^{N}$ is the membership relation:

$$
\begin{array}{rlrl}
i p p(p, g) & :=p^{\top} \mathrm{E} \cap g & i p m(p, g) & :=p^{\top} \mathrm{E} \cap \bar{g} \\
i m p(p, g) & :=p^{\top} \overline{\mathrm{E}} \cap g & i m m(p, g):=p^{\top} \overline{\mathrm{E}} \cap \bar{g}
\end{array}
$$

Then we have for all $X \in 2^{N}$ : If the $X$-column of $\mathrm{E}$ equals the inclination vector $i: N \leftrightarrow \mathbf{1}$, then we have that ipp $(p, g)_{\perp, X}$ holds iff $i \in I_{k}^{++}, \operatorname{ipm}(p, g)_{\perp, X}$ holds iff $i \in I_{k}^{+-}$, imp $(p, g)_{\perp, X}$ holds iff $i \in I_{k}^{-+}$, and $\operatorname{imm}(p, g)_{\perp, X}$ holds iff $i \in I_{k}^{--}$.

In other words, the row vector $\operatorname{ipp}(p, g)$ precisely designates those columns of the membership relation $\mathrm{E}$ which belong to the set $I_{k}^{++}$, and the remaining three row vectors do the same for the sets $I_{k}^{+-}, I_{k}^{-+}$and $I_{k}^{--}$, respectively.

\subsection{Computing the influence indices}

We assume a coalition $S$ of agents to be described by a column vector $s: N \leftrightarrow \mathbf{1}$, and an agent $j \in N$ to be described by a column point $p: N \leftrightarrow \mathbf{1}$. We compute the possibility influence index of $S$ on $j$. Since it is defined by means of the sizes of the sets $I_{S \rightarrow j}$ and $I_{S \rightarrow j}^{*}(B)$, we need to describe these sets within relation algebra. $I_{S \rightarrow j}$ and $I_{S \rightarrow j}^{*}(B)$ are subsets of $I_{S}$. In [7] the following is shown:

Theorem 6.4 Assume $s: N \leftrightarrow \mathbf{1}$ to be a description of the coalition $S \subseteq N$ and the row vector is $(s)$ of type $\left[\mathbf{1} \leftrightarrow 2^{N}\right]$ to be defined as

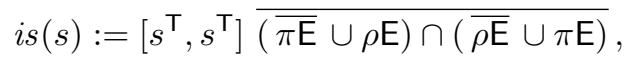

where $\mathrm{E}: N \leftrightarrow 2^{N}$ is the membership relation, and $\pi: N \times N \leftrightarrow N$ and $\rho$ : $N \times N \leftrightarrow N$ are the projection relations. Then we have for all $X \in 2^{N}$ : If the $X$-column of $\mathrm{E}$ equals the inclination vector $i: N \leftrightarrow \mathbf{1}$, then is $(s)_{\perp, X}$ holds iff $i \in I_{S}$.

Hence, the row vector is $(s)$ precisely designates those columns of the membership relation $\mathrm{E}$ which belong to the set $I_{S}$. Next, we deliver the relationalgebraic specification of the set $I_{S \rightarrow j}$, where $j \in N$ is described by the column point $p: N \leftrightarrow \mathbf{1}$. In [7] we prove the following theorem: 
Theorem 6.5 Assume $s: N \leftrightarrow \mathbf{1}$ describes the coalition $S \subseteq N$, the column point $p: N \leftrightarrow \mathbf{1}$ describes agent $j \in N$, the column point $q \subseteq s$ describes agent $k \in S$, and the row vector potinf $(s, p)$ of type $\left[\mathbf{1} \leftrightarrow 2^{N}\right]$ is defined by

$$
\operatorname{potinf}(s, p):=\left(\left(r \cup r^{\prime}\right) \cap \overline{r \cap r^{\prime}}\right) \operatorname{inj}\left(i s(s)^{\top}\right),
$$

where $r:=p^{\top} \mathrm{E} \operatorname{inj}\left(i s(s)^{\top}\right)^{\top}$ and $r^{\prime}:=q^{\top} \mathrm{E} \operatorname{inj}\left(i s(s)^{\top}\right)^{\top}$ with $\mathrm{E}: N \leftrightarrow 2^{N}$ as membership relation. Then we have for all $X \in 2^{N}$ : If the $X$-column of $\mathrm{E}$ equals the inclination vector $i: N \leftrightarrow \mathbf{1}$, then potinf $(s, p)_{\perp, X}$ holds iff $i \in I_{S \rightarrow j}$.

Hence, we relation-algebraically specify a row vector that precisely designates those columns of E which are inclination vectors of potential influence of $S$ on $j$.

To obtain a row vector $\inf (s, p, D)$ of type $\left[\mathbf{1} \leftrightarrow 2^{N}\right]$ that precisely designates those columns of the membership relation $\mathrm{E}: N \leftrightarrow 2^{N}$ which are inclination vectors of influence of $S$ on $j$, i.e., members of $I_{S \rightarrow j}^{*}(B)$, we use the equation

$$
I_{S \rightarrow j}^{*}(B)=I_{S \rightarrow j} \cap\left\{i \in I_{S} \mid(B i)_{j}=i_{S}\right\} .
$$

The relation-algebraic specification of $I_{S \rightarrow j}^{*}(B)$ is given by the row vector

$$
\inf (s, p, D):=\operatorname{potinf}(s, p) \cap \overline{\left(r \cup r^{\prime}\right) \cap \overline{r \cap r^{\prime}}} \operatorname{inj}\left(i s(s)^{\top}\right)
$$

with $r$ and $r^{\prime}$ given by $r:=p^{\top} \operatorname{Dvec}(D) \operatorname{inj}\left(i s(s)^{\top}\right)^{\top}$ and $r^{\prime}:=q^{\top} \operatorname{Einj}\left(i s(s)^{\top}\right)^{\top}$.

\subsection{Computing the sets of followers}

For modeling sets of followers we use column vectors. The relations $R$ and $Q$ column-wisely enumerate $I_{S}$ and $B\left(I_{S}\right)$, respectively, and the column point $q$ is used for specifying for $i \in I_{S}$ the specific Boolean value $i_{S}$. In [7] we show that:

Theorem 6.6 Assume $s: N \leftrightarrow \mathbf{1}$ to describe the coalition $S \subseteq N$, and the column point $q \subseteq s$ to describe some player $k \in S$. Furthermore, let $\mathrm{E}: N \leftrightarrow 2^{N}$ be the membership relation. If the column vector follow $(D, s)$ of type $[N \leftrightarrow \mathbf{1}]$ is defined as

$$
\text { follow }(D, s):=\operatorname{syq}\left(Q^{\top}, R^{\top} q\right)
$$

with relations $R:=\operatorname{E} \operatorname{inj}\left(i s(s)^{\top}\right)^{\top}$ and $Q:=\operatorname{Dvec}(D) \operatorname{inj}\left(i s(s)^{\top}\right)^{\top}$, then for all $j \in N$ we have follow $(D, s)_{j, \perp}$ iff $j \in F_{B}(S)$.

\section{Concluding remarks}

We have presented different measures of centrality that capture complementary aspects of a node's position in a network. As remarked in [19], the measures based on degree, closeness, and betweenness imply different "theories" of how centrality might affect group processes: centrality as activity, as independence, and as 
control. Despite this fact, all centrality measures should have some features in common, e.g., they should rank highest the most central node. As concluded in [19] all the three measures of network centrality agree in assigning the maximum centrality score to the star, and the minimum centrality score to a cycle and complete networks. Between these extremes, the three measures of network centrality may differ significantly in their rankings of networks. In a given application, one centrality measure or a combination of some measures might be more appropriate than another measure or a combination of measures.

Many centrality measures have not been discussed in this paper. A very interesting work is e.g., [1], where the intercentrality of a node in a network is investigated. Roughly speaking, it is the sum of the node's Bonacich centrality and its contribution to Bonacich centrality of other nodes. Apart from several sociological contributions to measuring centrality in social networks, also a game theoretic approach to centrality concepts is presented in the literature. For example, in [31] the authors propose a new definition of degree of centrality based on some extension of the Banzhaf index [2]. Also many works by Van den Brink and his co-authors deliver game theoretic measures of centrality in networks; see e.g. $[32,41,42,43]$.

Despite the existence of numerous centrality measures, as remarked in [12] most of the sociologically interesting processes are not covered by the major measures. For instance, there are no measures appropriate for infection and gossip processes. It seems therefore important to investigate centrality measures that could fill that gap.

It has been proved by numerous works (see e.g. [5, 6, 7, 8]) that the relationalgebraic approach to game theoretic problems is very appropriate and useful. There are still many more possibilities for combining relation algebra and RELVIEW to investigate and solve problems from Game Theory and Social Choice Theory. One of them might be an application of the tools in question to some centrality measures.

\section{References}

[1] Ballester, C., Calvo-Armengol, A., Zenou, Y.: Who's Who in Networks. Wanted: The Key Player. Econometrica 74, 1403-1417 (2006)

[2] Banzhaf, J.: Weighted Voting Doesn't Work: A Mathematical Analysis. Rutgers Law Review 19, 317-343 (1965)

[3] Behnke, R., Berghammer, R., Meyer, E., Schneider, P.: RelView - A System for Calculation with Relations and Relational Programming. In: Astesiano, E. (ed.) Fundamental Approaches to Software Engineering. LNCS, vol. 1382, pp. 318-321. Springer (1998)

[4] Berghammer, R., Karger, B. von, Ulke, C.: Relation-algebraic Analysis of Petri Nets with RelView. In: Margaria, T., Steffen, F. (eds.) Tools and Applications for the Construction and Analysis of Systems. LNCS, vol. 1055, pp. 49-69. Springer (1996)

[5] Berghammer, R., Rusinowska, A., De Swart, H.: Applying Relational Algebra and RelView to Coalition Formation. EJOR 178/2, 530-542 (2007) 
[6] Berghammer, R., Rusinowska, A., De Swart, H.: An Interdisciplinary Approach to Coalition Formation. EJOR 195, 487-496 (2009)

[7] Berghammer, R., Rusinowska, A., De Swart, H.: Applying Relational Algebra and RelView to Measures in a Social Network. EJOR 202, 182-195 (2010)

[8] Berghammer, R., Bolus, S., Rusinowska, A., De Swart, H.: A Relationalgebraic Approach to Simple Games. EJOR 210, 68-80 (2011)

[9] Berghammer, R., Schmidt, G., Winter, M.: RelView and Rath - Two Systems for Dealing with Relations. In: De Swart et al. (eds.), pp. 1-16 (2003)

[10] Bonacich, P.: Factoring and Weighting Approaches to Status Scores and Clique Identification. Journal of Mathematical Sociology 2, 113-120 (1972)

[11] Bonacich, P.: Power and Centrality: A Family of Measures. American Journal of Sociology 92, 1170-1182 (1987)

[12] Borgatti, S.P.: Centrality and Network Flow. Social Networks 27, 55-71 (2005)

[13] Brink, C., Kahl, W., Schmidt, G. (eds.): Relational Methods in Computer Science. Advances in Computing Science. Springer (1997)

[14] DeGroot, M.H.: Reaching a Consensus. Journal of the American Statistical Association 69, 118-121 (1974)

[15] DeMarzo, P., Vayanos, D., Zwiebel, J.: Persuasion Bias, Social Influence, and Unidimensional Opinions. Quarterly Journal of Economics 118, 909968 (2003)

[16] De Swart, H., Orlowska, E., Schmidt, G., Roubens, M. (eds.): Theory and Applications of Relational Structures as Knowledge Instruments. LNCS, vol. 2929, Springer (2003)

[17] De Swart, H., Orlowska, E., Schmidt, G., Roubens, M. (eds.): Theory and Applications of Relational Structures as Knowledge Instruments II. LNAI, vol. 4342, Springer (2006)

[18] Freeman, L.: A Set of Measures of Centrality Based on Betweenness. Sociometry 40, 35-41 (1977)

[19] Freeman, L.: Centrality in Social Networks: Conceptual Clarification. Social Networks 1, 215-239 (1979)

[20] Friedkin, N.E., Johnsen, E.C.: Social Influence and Opinions. Journal of Mathematical Sociology 15, 193-206 (1990)

[21] Friedkin, N.E., Johnsen, E.C.: Social Positions in Influence Networks. Social Networks 19, 209-222 (1997)

[22] Golub, B., Jackson, M.O.: Naïve Learning in Social Networks and the Wisdom of Crowds. American Economic Journal: Microeconomics 2(1), 112-149 (2010)

[23] Goyal, S.: Connections: An Introduction to the Economics of Networks. Princeton University Press (2007)

[24] Grabisch, M., Rusinowska, A.: Measuring Influence in Command Games. Social Choice and Welfare 33, 177-209 (2009)

[25] Grabisch, M., Rusinowska, A.: A Model of Influence in a Social Network. Theory and Decision 69(1), 69-96 (2010) 
[26] Grabisch, M., Rusinowska, A.: A Model of Influence with an Ordered Set of Possible Actions. Theory and Decision 69(4), 635-656 (2010)

[27] Grabisch, M., Rusinowska, A.: Different Approaches to Influence Based on Social Networks and Simple Games. In: Van Deemen and Rusinowska (eds.) Collective Decision Making: Views from Social Choice and Game Theory. Series Theory and Decision Library C, vol. 43, Springer-Verlag Berlin Heidelberg, pp. 185-209 (2010)

[28] Grabisch, M., Rusinowska, A.: A Model of Influence with a Continuum of Actions. GATE Working Paper, 2010-04 (2010)

[29] Grabisch, M., Rusinowska, A.: Iterating Influence Between Players in a Social Network. CES Working Paper, 2010.89 (2010)

[30] Grabisch, M., Rusinowska, A.: Influence Functions, Followers and Command Games. Games and Economic Behavior, DOI: 10.1016/j.geb.2010.06.003, forthcoming

[31] Grofman, B., Owen, G.: A Game Theoretic Approach to Measuring Degree of Centrality in Social Networks. Social Networks 4, 213-224 (1982)

[32] Hendrickx, R., Borm, P., Van den Brink, R., Owen, G.: The VL Control Measure for Symmetric Networks. Social Networks 31, 85-91 (2009)

[33] Hoede, C., Bakker, R.: A Theory of Decisional Power. Journal of Mathematical Sociology 8, 309-322 (1982)

[34] Hu, X., Shapley, L.S.: On Authority Distributions in Organizations: Controls. Games and Economic Behavior 45, 153-170 (2003)

[35] Hu, X., Shapley, L.S.: On Authority Distributions in Organizations: Equilibrium. Games and Economic Behavior 45, 132-152 (2003)

[36] Jackson, M.O.: Social and Economic Networks. Princeton University Press (2008)

[37] Katz, L.: A New Status Index Derived from Sociometric Analysis. Psychometrika 18, 39-43 (1953)

[38] Rusinowska, A.: Different Approaches to Influence in Social Networks. Invited tutorial at the COMSOC 2010 (Third International Workshop on Computational Social Choice, Düsseldorf), available at: http://ccc.cs.uniduesseldorf.de/COMSOC-2010/slides/invited-rusinowska.pdf

[39] Rusinowska, A., De Swart, H.: Generalizing and Modifying the HoedeBakker Index. In: De Swart et al. (eds.), pp. 60-88 (2006)

[40] Schmidt, G., Ströhlein, T.: Relations and Graphs, Discrete Mathematics for Computer Scientists. EATCS Monographs on Theoretical Computer Science, Springer (1993)

[41] Van den Brink, R., Gilles, R.: Measuring Domination in Directed Networks. Social Networks 22, 141-157 (2000)

[42] Van den Brink, R.: The Apex Power Measure for Directed Networks. Social Choice and Welfare 19, 845-867 (2002)

[43] Van den Brink, R., Borm, P., Hendrickx, R., Owen, G.: Characterization of the $\beta$ - and the Degree Network Power Measure. Theory and Decision 64, 519-536 (2008)

[44] Wasserman, S., Faust, K.: Social Network Analysis: Methods and Applications. Cambridge University Press, Cambridge (1994) 